\title{
CALCULATIONS OF NATIVE DEFECTS IN CUBIC AIN
}

\author{
I. Gorczyca* , A. Svane and N.E. Christensen
}

Institute of Physics and Astronomy, University of Aarhus, 8000 Aarhus C, Denmark

We have studied the electronic structure of native defects in cubic AlN. $\mathrm{N}$ and $\mathrm{Al}$ vacancies, antisites and interstitials are investigated in different charge states. We have performed first-principles calculations based on density-functional theory, using two methods. The first one is the Green-function technique based on the linear muffin-tin orbital method in the atomic-spheres approximation. Defects considered are all ideal substantial ones, i.e., no relaxation of the neighboring atoms is allowed for in this method. The results for aluminium vacancy and for nitrogen antisite are compared to the calculations using supercell approach and the full-potential linear muffin-tin orbital (the second method) with lattice relaxation included.

PACS numbers: 73.61.Ey, 71.55.Eq

\section{Introduction}

III-V nitrides are of great interest nowadays due to their properties important to applications [1] and to fundamental materials science. However, little is known about point defects in these materials. In this paper we concentrate on native point defects (vacancies, antisites, interstitials) in one of the most important representatives of nitrides - AlN. AlN can crystallize in two phases - cubic and wurtzite. We focus here on the cubic phase believing that there is no substantial difference in impurity level positions between the two phases and conclusions drawn from the present calculations can be applied to the wurtzite phase as well. A comparison of defect properties for wurtzite and cubic GaN made by Neugebauer and Van de Walle [2] showed only minor deviations in formation energy and atomic relaxations between these two hosts, and energy level positions remained practically the same. The only difference is a small split of the $t_{2}$ gap states related to the lower symmetry of the wurtzite structure compared to the cubic structure.

*Permanent address: High Pressure Research Center PAN, Sokołowska 29, 01-142 Warsaw, Poland. 


\section{Method}

To perform first-principles calculations we have used two methods based on linear muffin-tin orbital (LMTO) method. Both are using the local-density approximation (LDA) [3] to density-functional theory (DFT), by which exchange and correlation effects are accounted for by a simple local potential. The first method is the Green function technique [4]. The second one is the full-potential LMTO in supercell geometry.

In the Green-function method the valence electronic structure of the impurity atom is obtained from the Green function $G$, which is found by solving the Dyson equation with $G_{0}$ - the Green function of the pure crystal host, and $\Delta V$ - the perturbation due to the impurity. In the LMTO method the host Green function is calculated from the band structure of the pure crystal within the atomic sphere approximation (ASA), i.e., the crystal volume is approximated by slightly overlapping atom centered spheres, inside which the potential is made spherically symmetric. Additional "empty" spheres are introduced at the tetrahedral interstitial sites [5]. In this paper we choose all spheres to have the same size determined by the experimental host lattice constant. In the calculations we have used the "standard" basis set which includes partial waves of $s, p$, and $d$ character on each atomic and interstitial site to give a total of 36 LMTO orbitals per unit cell. The unperturbed Green function is calculated with the tetrahedron method using $95 k$ points in the irreducible wedge of the Brillouin zone.

In the LDA approximation the fundamental gaps of semiconductors generally are computed $50-100 \%$ too small. This has a significant impact on defect calculations, in particular on the energy level positions of bound states in the gap. To overcome this problem we have shifted rigidly the conduction bands upwards to match the experimental minimum rap (the "scissors operator").

The LMTO Green function method describes a single impurity in an infinite crystal. Consequently, defect level positions are determined exactly, but relaxations of the neighboring atomic positions are not allowed for.

The results for aluminium vacancy and for nitrogen antisite are compared to the calculations using supercell approach and full-potential LMTO (the second method) with lattice relaxation included. In this case calculations are carried out in a 32-atom supercell. To find the minimum energy configuration for the given defect, we allowed all the surrounding atoms to relax, fixing the coordinates of the defect atom, and presuming the same symmetry of the defect as in the unrelaxed case. The finite size of the supercell causes the impurity states to broaden, and the level positions must be determined from the centers of the impurity bands, which leads to some uncertainty.

\section{Positions of the defect levels}

The energy gap of AlN in the zinc-blende structure as calculated by us is equal to $6.1 \mathrm{eV}$. The resulting energy levels of native defects in AlN are shown in Fig. 1. The vacancies, the anstisite defects and isolated interstitial atoms at two inequivalent tetrahedral positions are considered. For the aluminum vacancy, $\mathrm{V}_{\mathrm{Al}}$, and for the aluminum antisite, $\mathrm{Al}_{\mathrm{N}}$, both neutral and charged states are considered. 


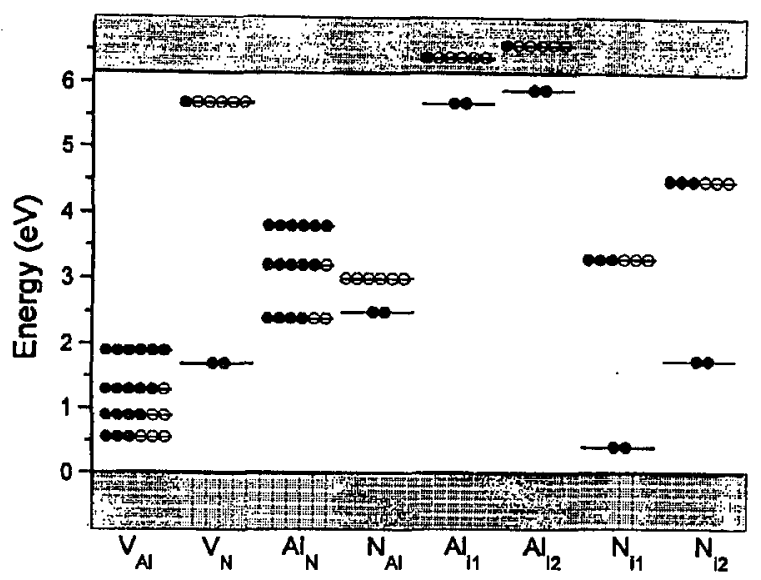

Fig. 1. Energy levels of native point defects in AlN.

$\mathrm{V}_{\mathrm{Al}}$ in the neutral charge state is a triple acceptor, with a level close to the valence band, which can be filled with three more electrons, introducing a set of levels ranging from $0.6 \mathrm{eV}$ to $1.9 \mathrm{eV}$ above the valence band edge. According to the present calculations the $a_{1}$ ( $s$-like) state of $V_{N}$ lies at $1.7 \mathrm{eV}$ above the valence band edge, whereas the $t_{2}$ ( $p$-like) state, occupied by one electron, lies at $5.7 \mathrm{eV}$ above valence band.

Aluminum antisite, $\mathrm{Al}_{\mathrm{N}}$, introduces a set of levels in the band gap, ranging from $2.4 \mathrm{eV}$ to $3.8 \mathrm{eV}$ above the valence band edge. For the neutral charge state the triplet state in the band gap (situated at $2.4 \mathrm{eV}$ ) is occupied with four electrons (double acceptor). The neutral nitrogen antisite defect, $\mathrm{N}_{\mathrm{Al}}$, introduces a doubly occupied $a_{1}$ state at $2.5 \mathrm{eV}$ above the valence band maximum and an empty triplet at $3.0 \mathrm{eV}$.

For substitutional defects, the local topologies of the zinc-blende and wurtzite lattices are the same, since in both cases each atom has four first neighbors with tetrahedral symmetry. This is why the electronic structures of vacancies and antisites in both structures are very similar. The difference between the two structures begins at the second shell of neighbors and consequently, the local topologies of interstitials are quite different in the two lattices. In zinc-blende crystals there are two interstitial sites with tetrahedral symmetry, surrounded by four cations ( $i 1$ in Fig. 1) or four anions ( $i 2$ ), respectively. In our calculations for the zinc-blende structure we have found that the defect states corresponding to positions (i1) and (i2) of ar, interstitial aluminum atom are very close, giving rise to a shallow $s$-state at $5.7 \mathrm{e}$ and $5.9 \mathrm{eV}$ above the valence band edge, respectively.

For the $\mathrm{N}$ interstitial the $s$-states are lying at $0.4 \mathrm{eV}(i 1)$ and at $1.8 \mathrm{eV}(i 2)$ above valence band, whereas $t_{2}$ states, occupied by three electrons, are lying at $1.4 \mathrm{eV}$ and $1.9 \mathrm{eV}$ above the valence band maximum, for positions (i1) and (i2), respectively. 


\section{Lattice relaxation}

The supercell calculations by using full-potential LMTO [6] were performed for aluminum vacancy, $\mathrm{V}_{\mathrm{Al}}$, and for nitrogen antisite, $\mathrm{N}_{\mathrm{Al}}$. Applying this method we were looking mainly at the atomic geometry. In both cases we have found the outward relaxation around defect. For $\mathrm{V}_{\mathrm{Al}}$ the bond distance to the nearest neighbors is increased by $5.4 \%$, i.e., from the ideal value of $1.89 \AA$ to $1.99 \AA$. Looking at the defect level position, we get about $0.5 \mathrm{eV}$ (center of the impurity band) for the neutral charge state without relaxation, in good agreement with the result from the Green-function calculations $(0.6 \mathrm{eV})$. The relaxation induces a downward energy shift of about $0.2 \mathrm{eV}$. For $\mathrm{N}_{\mathrm{Al}}$ the outward displacement of the nearest neighbor nitrogen atoms is $1 \%$, i.e., bond length between defect and surrounding atoms is now $1.91 \AA$. The unrelaxed level position is about $2 \mathrm{eV}$, somewhat lower than that one obtained by Green-function method $(2.4 \mathrm{eV})$, and practically does not change when relaxation is included.

Comparing the above results with the supercell calculations for GaN made by pseudopotential method $[7,8]$, we can see that the relaxation around defect is very similar in the case of cation vacancy (for $\mathrm{V}_{\mathrm{Ga}}$ in $\mathrm{GaN}$ - the outward displacement of the nearest atoms is also $0.1 \AA[8]$ ). In the case of $\mathrm{N}_{\mathrm{Ga}}$ the bond distance to the nearest neighbors is reduced by $29 \%$ [7], while, as results from the present paper, for $\mathrm{N}_{\mathrm{Al}}$ the relaxation is outward and small. The above drastic difference can be explained by the fact that relaxing all the surrounding atoms we fix the coordinates of the defect atom. The relaxation of the defect atom position will be the next step of our calculations.

\section{Summary}

We have calculated the energy positions of all native point defects in cubic AlN. Comparing the present results for AlN with the earlier calculations for GaN [9], we can see that there are very similar tendencies in the positions of vacancies and antisites in GaN and AlN, although the absolute values are different, mainly due to the different values of the energy gaps. Looking at atomic geometry we have found outward lattice relaxation around $\mathrm{V}_{\mathrm{Al}}$, very similar as in the case of $V_{G a}[7]$, and very small outward relaxation in the case of $N_{A l}$.

This work was partially supported by the Committee for Scientific Research grant No. 2 P03B 17810.

\section{References}

[1] B.G. Levi, Physics Today 49, 18 (1996).

[2] J. Neugebauer, C.G. Van de Walle, Phys. Rev. B 50, 8067 (1994).

[3] R.O. Jones, O. Gunnarsson, Rev. Mod. Phys. 61, 681 (1989).

[4] O. Gunnarsson, O. Jepsen, O.K. Andersen, Phys. Rev. B 27, 7144 (1983).

[5] D. Glötzel, B. Segall, O.K. Andersen, Solid State Commun. 36, 403 (1980).

[6] M. Methfessel, Phys. Rev. B 38, 1537 (1988).

[7] P. Bogusławski, E.L. Briggs, J. Bernholc, Phys. Rev. B 51, 17255 (1995).

[8] J. Neugebauer, C.G. Van de Walle, Mater. Res. Soc. Symp. Proc. 339, 678 (1994).

[9] I. Gorczyca, A. Svane, N.E. Christensen, Acta Phys. Pol. A 88, 723 (1995). 